\title{
EL MANEJO DE LA INFORMACIÓN EN CUIDADOS PALIATIVOS
}

\author{
THE INFORMATION MANAGEMENT IN PALLIATIVE CARE
}

\author{
Yolanda Romero Rodríguez, Fátima López Tapia, José Martín de Rosales Martínez, $M^{a}$ \\ Ángeles Martin Sánchez, Juan Romero Cotelo y Rafael Galvez Mateos.
}

Unidad Cuidados Paliativos Hospital Virgen de las Nieves, Granada.

Resumen

Según la Ley de Autonomía del paciente (Ley 41/2002, del 14 de Noviembre), es el paciente quien tiene derecho a ser informado y el titular de la información que reciba. Se ha elaborado una entrevista estructurada que se ha administrado de forma aleatoria a los familiares de los pacientes incluidos en el proceso asistencial Unidad de Cuidados Paliativos (UCP) del Hospital Virgen de las Nieves de Granada, para evaluar el grado de conocimiento sobre esta ley. Además de evaluar el nivel de conocimiento que el paciente dispone sobre su diagnóstico/pronóstico. Los datos obtenidos en este estudio contrastan con esta ley, donde menos de la mitad de los pacientes, según sus familiares, conocen su enfermedad y una cifra inferior la cuarta parte está informado su pronóstico. Asimismo, la mayoría de los familiares de pacientes avanzados se muestran en contra de la ley de Autonomía del Paciente. Este hecho obliga a que los profesionales nos esforcemos más en lograr un proceso comunicativo que acerque al paciente a su realidad y acompañamiento en su última fase de la vida.

Palabras clave: Cuidados Paliativos; confidencialidad; comunicación.

\section{Abstract}

According to the patient's autonomy law (Law 41/2002, of November 14), is the patient who has the right to be informed and the owner of the information it receives. It has developed a structured interview was administered randomly to the relatives of the patients in the care process Palliative Care Unit (PCU) of the Virgen de las Nieves Hospital of Granada, to assess the level of awareness about this law. In addition to assessing the level of knowledge that the patient has about their diagnosis / prognosis. The data obtained in this study contrast with the Act, where less than half of patients, according to their families, know their disease and a lower figure is reported fourth of its forecast. Also, most advanced patient relatives are against the law of patient autonomy. This requires that more professionals we strive to achieve a communicative process that brings the patient to his reality and support in their last phase of life.

Key words: Palliative care, confidentiality, communication.

Correspondencia:

Yolanda Romero Rodríguez

Unidad del Dolor y Cuidados Paliativos. Hospital Virgen de las Nieves.

Avenida de las Fuerzas Armadas $\mathrm{s} / \mathrm{n}$

18010 Granada

E-mail: yolandaromerodriguez@gmail.com 


\section{INTRODUCCIÓN}

La progresión de una enfermedad crónica, el diagnóstico de una enfermedad incurable, la constatación del empeoramiento de la salud, son algunas de las múltiples situaciones que exponen a la persona y a sus familiares y allegados a una situación amenazante y difícil. Desde distintos ámbitos del conocimiento y también por parte de la sociedad se reclama, cada vez con más intensidad, la necesidad de atender profesional y humanamente a las personas en la etapa final de su vida.

La forma de afrontar estos fenómenos emocionales y cognitivos asociados a la cercanía de la propia muerte o de la muerte de un ser querido depende de los recursos personales con los que el individuo cuenta, del apoyo de su familia y del equipo de atención con que cuente, todo ello enmarcado en un contexto social-cultural ${ }^{(1)}$.

En la sociedad actual priman lo más básico e individual, la poca tolerancia a la frustración y se cataloga el sufrimiento como algo anormal, fomentándose una posición de negatividad y constante evasión del mismo. La muerte forma parte de la vida y morir constituye el acto final de la biografía personal de cada ser humano, y no puede ser separada de aquella como algo distinto (1).

Los profesionales sanitarios desempeñan un papel fundamental en la mejora de la calidad de vida de los pacientes durante toda la etapa de su enfermedad, pero especialmente cuando se encuentra en fase avanzada o terminal. Por ello, tal como señala Barreto $(2003)^{(2)}$, es totalmente necesaria la atención integral de estos pacientes en los aspectos físicos, psicológicos, afectivos y espirituales, con una comunicación adecuada que de respuesta a sus dudas y temores.

El ideal de la buena muerte se ha ido concretando durante los últimos 25 años como derecho, derecho a la muerte dig- na. En su formulación originaria haría referencia a que una exigencia ética debería de ser reconocida. Este derecho, aunque no está recogido en las declaraciones o pactos sobre derechos humanos, existe un debate importante acerca de poder considerarlo un nuevo derecho humano de tercera generación ${ }^{(3)}$.

Posiblemente el problema no esté tanto en el concepto formal, sino esté en el miedo a los contenidos que deban de incluirse en él. Y lo más difícil sea el decidir cómo y cuándo desea uno morir, por tanto puede decirse que existe un acuerdo en el plano ético- jurídico en que el derecho a la dignidad en el proceso de muerte implica el derecho a:

- Confidencialidad de sus datos.

- Participación activa en toma de decisiones.

Se ha hecho necesaria la regulación de los derechos concernientes en la relación médico-paciente, desde la objetividad de una Ley que cubra las controversias que puedan aparecer en el ejercicio de la actividad médico-sanitaria en la que el paciente interviene de forma activa y directa. En mayo de 2003 entró en vigor la Ley Básica Reguladora de la Autonomía del Paciente, y de Derechos y Obligaciones en Materia de Información y Documentación Clínica (Ley 41/2002, del 14 de Noviembre) (LAP). Con ella quedan normalizados los objetivos de la información y la forma de comunicación con los pacientes, la privacidad y capacidad de decisión del individuo en cuestiones relativas a su salud y enfermedad, su participación en la toma de decisiones clínica y el proceso de actuación cuando el paciente carece de suficiente capacidad de decisión ${ }^{(4)}$.

Se pone de manifiesto la "importancia que tienen los derechos de los pacientes como eje básico de las relaciones clínicoasistenciales". De esta manera, es el médico el encargado de proporcionar al paciente la información "adecuada" sobre la 
naturaleza de su enfermedad, su proceso diagnóstico y terapéutico, para que pueda tomar decisiones de manera libre y voluntaria. Información adecuada es aquella información expresada de forma comprensible a las capacidades de cada paciente. Podrán ser informados los familiares de los pacientes en la medida que estos lo permitan, o en el caso de que el paciente, por su estado físico o psíquico, carezca de capacidad para entender la situación ${ }^{(4)}$.

Este derecho del paciente a la información también puede verse limitado siempre que, por razones objetivas, el conocimiento por parte del paciente de su situación pueda perjudicar su salud de manera grave. En este caso el médico podría actuar profesionalmente sin informar con anterioridad al paciente. De la misma forma, toda persona tiene derecho a no ser informada, si así lo desea.

Por otra parte, existe un documento de registro de voluntades anticipadas. El objetivo principal de las Voluntades Anticipadas, según establece la Ley 5/2003 del 9 de Octubre ${ }^{(5)}$ es asegurar que, cuando el paciente haya perdido la capacidad para participar en la toma de decisiones, la atención sanitaria se realice conforme a las preferencias que hayan expresado previamente. En el Documento de Voluntades Anticipadas se pueden plasmar por escrito:

1. La expresión de los principios vitales y las opciones personales respecto a los momentos finales de la vida $u$ otras situaciones de grave limitación física o psíquica en los que se establece una jerarquía de valores $y$, en consecuencia, los criterios que deben orientar cualquier decisión que se deba tomar en el ámbito sanitario. También pueden especificarse otras consideraciones como, por ejemplo, la elección del lugar (hospital, domicilio) donde se desea recibir los cuidados en el final de la vida, la voluntad de ser donante de órganos, el deseo de recibir asistencia religiosa o no, si se es contrario a que se practique au- topsia, si se desea donar el cuerpo para estudios anatómicos, etc.

2. Las situaciones sanitarias concretas en que se quiere que se tengan en cuenta la aceptación o rechazo de determinados tratamientos o cuidados, sobre todo cuando se tiene información de las probabilidades evolutivas.

3. Instrucciones y límites a las actuaciones médicas ante las situaciones previstas, es decir, qué quisiera y qué no quisiera la persona respecto a tratamientos y cuidados en caso de situaciones irreversibles para la vida. Así, por ejemplo, se puede solicitar que no sean aplicadas (o se retiren, si ya se han aplicado) medidas de soporte vital tales como: reanimación cardiopulmonar, diálisis, conexión a un respirador, nutrición e hidratación artificiales que impliquen prolongación de la vida innecesariamente (huyendo del encarnizamiento terapéutico) y siempre que no vaya contra el ordenamiento jurídico vigente.

4. La designación de un representante para que actúe como interlocutor válido ante el equipo sanitario en el caso de que el paciente no pueda expresar su voluntad y para que lo sustituya en la interpretación y cumplimiento de las instrucciones.

En definitiva, las voluntades anticipadas se enmarcan en la planificación de cuidados, entendida como un proceso positivo de reflexión e implicación de los ciudadanos y ciudadanas en las decisiones relativas a su salud, de acuerdo con sus valores personales en el marco de la relación médico-paciente-familia, es decir, que una buena práctica médica ya no lo es si no respeta la voluntad de la persona enferma.

Los objetivos del equipo multidisciplinar que trabaja con el enfermo y su familia deben converger en facilitar la adaptación de ambos a esta difícil situación, promoviendo el máximo bienestar posible y amortiguando el sufrimiento.

Esta situación exige un proceso comunicativo con el familiar y el paciente, 
con estrategias contrastadas, que logre que se sienta acogido y cuidado. Es decir, que la obligación moral del profesional debe establecer con el paciente y la familia procesos deliberativos que realmente le ayuden en la toma de decisiones $^{(6)}$.

Si bien a corto plazo evitar temas difíciles, ocultar información a la persona moribunda, o pedirle al médico que no hable delante de él sobre su pronóstico, alivia a los familiares; a largo plazo y conforme las dificultades avancen, el enfermo termina percibiendo su propio deterioro ${ }^{(1)}$. Estos intentos por protegerle pueden llegar a generar mayor tensión en la familia, pueden resultar en un bloqueo en a comunicación y en un distanciamiento emocional con el enfermo. En definitiva, se verá que lo problemático son los intentos por huir del sufrimiento, por no pensar o sentir, por ahuyentar los recuerdos, la lucha contra el miedo, las dudas...

El objetivo de este trabajo es conocer la información y la valoración sobre la LAP y de la Ley de Voluntades Anticipadas que poseen los familiares de pacientes incluidos en el proceso asistencial de cuidados paliativos de la Unidad de Cuidados Paliativos (UCP) del Hospital Virgen de las Nieves de Granada.

\section{MATERIAL Y MÉTODO}

Se ha elaborado una entrevista estructurada que se ha administrado de forma aleatoria a los familiares de los pacientes incluidos en el proceso asistencial de la UCP. La entrevista consta de 34 ítems con varias alternativas de respuesta: 8 ítems que recogen información demográfica, 6 ítems referidos a la enfermedad del paciente, 12 ítems acerca de la información de la que el paciente dispone sobre su diagnóstico/pronóstico 2 ítem referidos a cómo el personal sanitario di la información médica, 3 ítem referidos al conocimiento y el acuerdo con la LAP 2 ítems referidos al conocimiento que se tiene de su testamento vital y 1 ítem referido a la importancia sobre el apoyo psicológico.

La entrevista ha sido realizada por los profesionales de la UCP a 49 familiares de pacientes que fueron incluidos en el proceso asistencial de Cuidados Paliativos, entre Octubre 2009 y Febrero de 2010. Una vez terminada la consulta médica, se les informó a los familiares de los pacientes sobre las dos leyes anteriormente citadas y se les administró la entrevista durante 15 minutos aproximadamente. Los datos obtenidos han sido informatizados y se han analizado estadísticos descriptivos con el programa SPSS 15.0

\section{RESULTADOS}

Los 49 familiares que acudieron a consulta con el paciente participaron de forma voluntaria e individual. Respecto a los análisis descriptivos de la muestra observamos que la edad varía entre 24 y 78 años, con una media de 48,8 (desviación típica $=13,4)$. En cuanto al género, un $83,7 \%$ son mujeres y un $16,3 \%$ son hombres.

El perfil general de estos familiares que acuden a consulta con el paciente es el siguiente:

Son hijos/as del paciente $(65,3 \%)$, pareja $(10 \%)$ con un nivel educativo correspondiente a estudios primarios $(32,7 \%)$, casados $(71,4 \%)$, solteros $(20,4 \%)$ procedentes de Granada (área rural $=51 \%$, distrito urbano $=40,8 \%$ ).

Respecto a la situación laboral familiar, son trabajadores/as en activo (38,8 $\%)$, amas de casa $(26,5 \%)$, pensionistas $(12,2 \%)$, parados $(6,1 \%)$, estudiantes $(4 \%)$, jubilado $(8,2 \%)$.

El cáncer más predominante de estos pacientes paliativos fue el de pulmón $(22,4$ $\%)$, seguido del cáncer gástrico $(12,2 \%)$. En la mayoría de los casos existe un estado 
de neoplasia diseminada $(71,4 \%)$. Aunque la media de supervivencia de estos enfermos desde que conocen su diagnóstico hasta la fecha de óbito es de 554,24 días, en la UCP están una media de 104 días (mediana $=68$ ).

Respecto al conocimiento que el paciente tiene de su enfermedad se revela lo siguiente: un $49 \%$ de los pacientes conoce su enfermedad, y de ellos, solo el $50 \%$ tiene una idea de su pronóstico, es decir, apenas el $24 \%$ tiene toda la información. Hay un $69,4 \%$ de los pacientes que no hablan de su enfermedad con sus familiares.

En la mayoría de los casos $(86,1 \%)$ ha sido el médico quien comunicó el diagnóstico oncológico al familiar entrevistado, a la mitad de ellos informó también al paciente y en pocos casos el familiar informado lo comunicó después a su allegado $(4 \%)$.
En referencia sobre la actuación de la LAP, el 61,2\% de los familiares no es partidario de que se informe primero al paciente sobre la enfermedad; un $69,4 \%$ piensa que la información debe darse a los familiares en primer lugar. Incluso, gran parte de estos familiares $(65 \%)$ opina que no es bueno que el paciente conozca la enfermedad. (figura 1)

En cuanto al recurso de voluntades anticipadas, un 71,4\% de los familiares cree que no es conveniente que se le informe al paciente de que existe este documento (figura 2). Menos de la mitad de estos familiares $(46,9 \%)$ conocen la existencia del testamento vital.

Los familiares $(71,4 \%)$ afirman que sería bueno el apoyo psicológico durante todo el proceso de enfermedad, desde el momento que se les da la información diagnóstica.

Figura 1. Está de acuerdo con la LAP

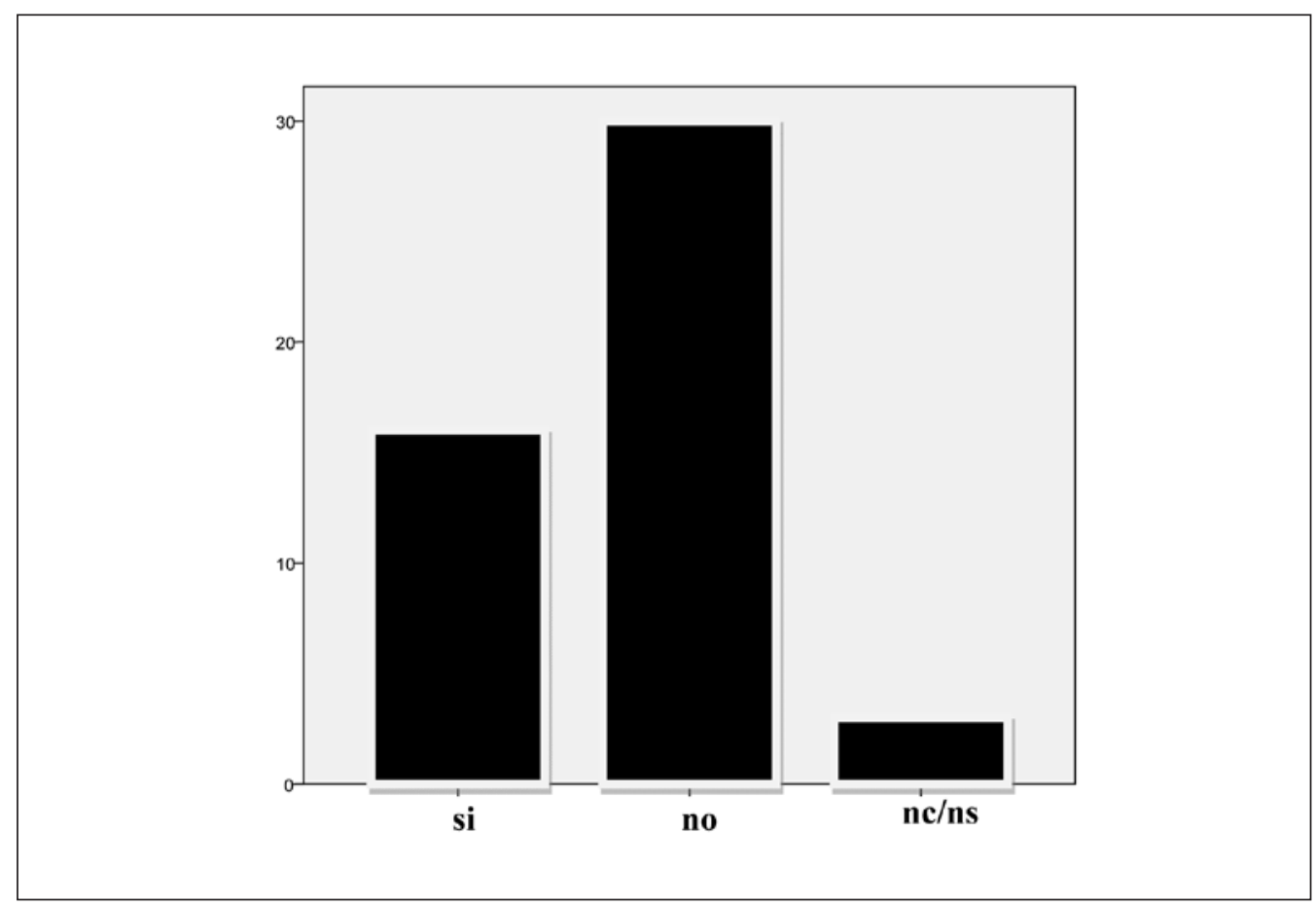


Figura 2.

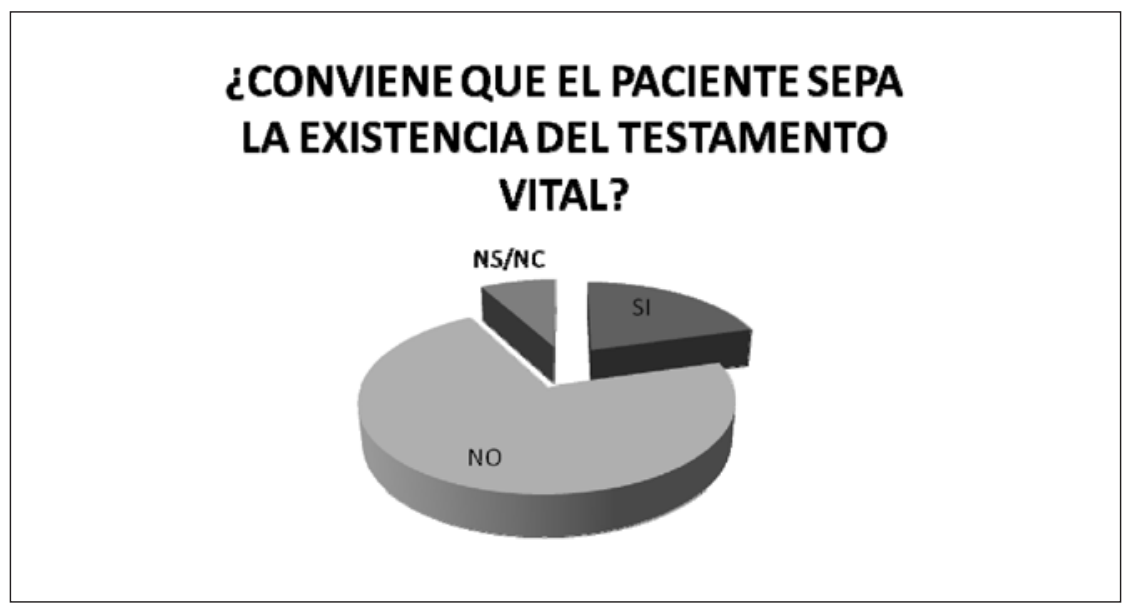

\section{DISCUSIÓN}

Según la LAP, es el paciente quien tiene derecho a ser informado y el titular de la información que reciba. Los datos obtenidos en este estudio contrastan con esta ley. Menos de la mitad de los pacientes, según sus familiares, conocen su enfermedad y sólo un 16\% está informado del diagnóstico y del pronóstico.

En un estudio sobre la comunicación del diagnóstico de cáncer en España en el que se recogen los resultados de distintas investigaciones. Centeno et al..$^{(7)}$ informan que el $75 \%$ de los pacientes informados hablaba claramente de su enfermedad y de sus consecuencias con sus familiares, mientras que solamente el $25 \%$ de los no informados hacía lo mismo. Los pacientes informados identificaban con más nitidez al médico de referencia, estaban más satisfechos de su relación con él y comprendían mejor las explicaciones recibidas.

En un estudio posterior, Centeno y Nu$\tilde{n} z^{(8)}$ afirman que en nuestro medio un 40$70 \%$ de los enfermos con cáncer conocen la naturaleza maligna de su enfermedad aun cuando sólo un $25-50 \%$ han sido informados de ello. Por esto mantiene que "el enfermo sabe habitualmente bastante más de lo que se le ha dicho" probablemente porque tiene otras fuentes de información (entre ellas su propio organismo). Conforme el tiempo avanza, es lógico que el deterioro físico que va presentando progresivamente le sirve al paciente como un buen indicador del pronóstico negativo de su enfermedad. Este hecho obliga a que los profesionales nos esforcemos más en lograr un proceso comunicativo que acerque al paciente a su realidad ya que cuando la información no proviene del equipo que atiende al enfermo existe el riesgo de que surja en éste la desconfianza, la sensación de engaño y el conflicto.

El enfermo también tiene derecho a rechazar la información diagnóstica y/o pronóstica, y el apoyo solidario y efectivo de profesionales y voluntarios; pero para que haya rechazo tiene que haber oferta. $Y$ esta oferta es una responsabilidad ética de todos los profesionales desde el momento en que empiezan a intervenir en su atención ${ }^{(9)}$. Según la LAP, los usuarios del sistema sanitario tienen el derecho a no ser informados. Según los resultados presentes, más de la mitad de los pacientes $(69,4 \%)$ no preguntan sobre su enfermedad a sus 
familiares (figura 3). Sin embargo, queda por analizar si estos pacientes realmente no quieren saber su diagnóstico o si lo que ocurre es que no se le da opción a preguntar.

Según Barbero, el problema de la información parece ser un dilema entre los principios éticos de autonomía y maleficencia. Tan maleficente puede ser el encarnizamiento informativo (informar de un diagnóstico o pronóstico negativos a un paciente que no quiere saber), como la conspiración del silencio frente a un paciente que desea ser informado.

Aquí aparece, el debate entre la dialéctica entre técnica y ética, entre comunicación y deliberación. La información es un acto clínico -esto está admitido en la práctica y además recogido en la ley-, desafortunadamente es probable que todavía falten años para que la legislación recoja que también la comunicación terapéutica y la deliberación.

Esto significa que la obligación moral del profesional no estriba únicamente en comunicarse adecuadamente con el paciente desde un punto de vista terapéuti- co, sino que también debe establecer con él procesos deliberativos que realmente le ayuden en la toma de decisiones. La comunicación terapéutica exige adaptarte al otro, a su realidad, a sus sesgos cognitivos, a sus miedos, y también supone asumir el riesgo de ser uno mismo, porque en la comunicación también entramos nosotros desde nuestras ideas, nuestros afectos $y$ nuestros valores.

En este sentido la comunicación, que pretende ser una herramienta básica para la posterior deliberación moral, tiene algunas claves que conviene recordar: que hay que explorar permanentemente; no dar nada por supuesto, hay que identificar y explorar las preocupaciones que el paciente subjetivamente puede estar viviendo no sólo los problemas que el profesional objetiva. Hay que fomentar la actitud de la empatía y su correlato, no solo que el otro se sienta escuchado, sino también que pueda seguir explorando su mundo interno, sin miedo a adentrarse en él, para posteriormente ir tomando decisiones. Se debe de actuar desde la congruencia o autenticidad, con todas estas claves ayu-

Figura 3.

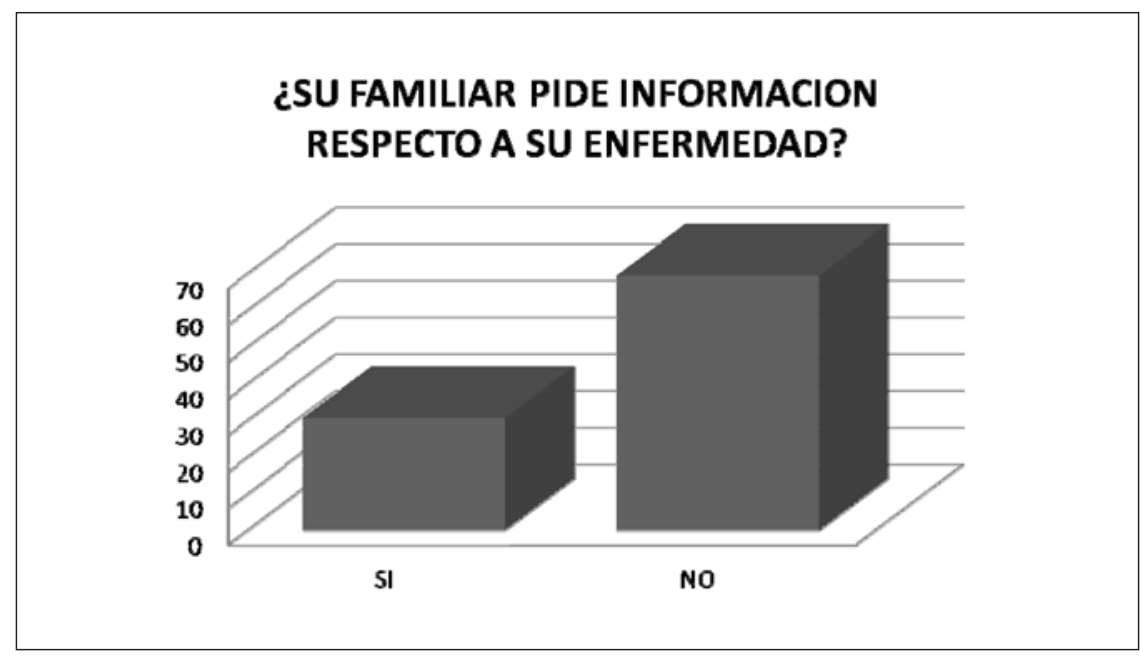


dara a propiciar la creación de un vínculo terapéutico.

La importancia de este estudio preliminar es que este cuestionario debería pasarse a los familiares en la valoración inicial al incluirlo en el proceso de cuidados paliativos, permitiéndonos conocer:

- La situación de la información y actitud del paciente sobre su diagnóstico y sobre todo de su pronóstico.

- Actitud de la familia sobre la información que debe darse al paciente.

Este conocimiento permite el trabajo posterior que deberá realizar un equipo especializado en este contexto, preferentemente un equipo de apoyo psicosocial (EAPS) que permitirá, entre otras cuestiones como la reducción de la conspiración del silencio, la facilitación al paciente la expresión de sus dudas y preocupaciones, fomentar y promover que el paciente realice el testamento vital y ayudar al equipo profesional sanitario facilitando estrategias de adaptación.

La comunicación y la deliberación es un arte que debe de aprenderse y usarse para una mayor satisfacción de los pacientes al ser escuchados, al percibir un ambiente de confianza y mayor sinceridad, un mejor conocimiento de su enfermedad o a un respeto hacia su negación adaptativa de su proceso informativo.

\section{REFERENCIAS BIBLIOGRÁFICAS}

1. Páez M. Cuidados psicológicos al final de la vida. Adiós 2009;74:20-5.

2. Barreto MP. Psicología y fin de la vida. Psicooncología 2003; 1:135-46.

3. Simón P. Alarcos FJ. Ética y muerte digna. Junta de Andalucía. Consejería de Salud, 2008.

4. Boletín Oficial del Estado. Ley 41/2002, básica reguladora de la autonomía del paciente y de derechos y obligaciones en materia de información y documentación clínica. BOE núm 274, de 15 de noviembre de 2002.p. 40126-32.

5. Boletín Oficial Junta de Andalucía. Ley 5/2003, Declaración del voluntad vital anticipada. BOJA, núm. 279, de 9 de octubre de 2003.

6. Montesinos F, Páez M. Vivir hoy el duelo. Adiós 2009;76: 6-9.

7. Centeno C, Núñez JM. Questioning diagnosis disclosure in terminal cancer patients: A prospective study evaluating patient's responses. Palliat Med 1994;8:39-44.

8. Centeno C, Núñez JM. Estudios sobre la comunicación del diagnóstico de cáncer en España. Med Clin (Barc) 1998;110:74450.

9. Barbero, J. El derecho del paciente a la información: el arte de comunicar. An Sist Sanit. Navar 2006;29:19-27. 